\title{
Formal Method of Structural-Logical Identification of Functional Model of Subject Area Polycubic Data Matrix
}

\section{Gennady Kulikov, Vyacheslav Antonov, Almira Fahrullina, Ludmila Rodionova, Ansaf Abdulnagimov, Maria Shilina}

Ufa State Aviation Technical University

Faculty of Computer Science and Robotics

K. Marx str, 12, 450008 Ufa, Russia

e-mail: kulikov.gg@ net.ugatu.su, antonov.vv@ net.ugatu.su, fahrullina.almira@net.ugatu.su, rodionova.lyudmila@net.ugatu.su, abdulnagimov.ai@net.ugatu.su, shilina.ma@net.ugatu.su

\footnotetext{
Abstract: This work proposes a technique of presenting a system model of a subject area in the form of a category of processes. The rule of synthesis of self-organizing structure of process models is proposed. It is shown that the sequence of process decomposition compositions has the property of fractal transformations (self-like). The core of these transformations are commutative triangles that define the structure of interacting processes in composition-decomposition. Such structures have been shown to retain the properties of self-organization in accordance with the laws of the required diversity of the EShBI. The possibility of building a number of identical formal algorithms of control of integrity of the structure of initial processes, in case of violation of which "external" control is required, is shown. The proposed transformation is demonstrated by the example of synthesis of the self-organizing process of the agreement, the architecture of which is defined in ISO/IEC 15288 standard "System Engineering." Polycubic data organization for a software system is proposed herein.
}

Keywords: software system; category theory; subject area; structural analysis and synthesis; formal model; system engineering standards; data processing and transmission; fractal

\section{Introduction}

At present, automated processing, transfer and use of personnel reserve data using software allows the enterprise not to find itself in a situation of shortage of personnel, upon dismissal or transfer of employees, which significantly increases the level of competitiveness of the organization. In these cases, the tool for 
improving the processing, transmission and use of personnel reserve data is intelligent content, which allows to formalize and structure data of enterprise specialists forming this reserve for further multidimensional analysis of data.

Intellectual content in this article refers to a set of information objects, data of human capital of an enterprise, their connections, characteristics, parameters preserving knowledge about the subject area under study.

Human capital is the amount of accumulated knowledge and skills, motivation and energy that can be used, for a certain period of time, to realize the objectives of the production of goods and services [1].

The filling of intelligent content forms data and knowledge of the personnel reserve of the enterprise. In turn, the personnel reserve is a group of data on specialists potentially seeking a leadership position, passed competitive selection and individual training [2].

At the same time, in the process of forming intelligent content there is a problem related to the use of two interrelated business processes of personnel reserve and personnel agency (for example, educational institutions). Considering the information objects of two interrelated business processes (on the example of a personnel reserve and a personnel agency), it is possible to use the standard ISO/IEC 15288 "System Engineering" most common in system engineering, which allows to formalize and structure intelligent content and relations between the objects in question on the example of agreement processes [3].

It is possible to solve the current theoretical and practical problem related to the creation of a fully decentralized platform on which business processes are carried out. Namely, the application of Blockchain technology, which allows to improve business processes, as well as to create a single language of communication. And, as a result, the complex problem of big data analytics, categories of information objects - software modules, ensuring the functioning of real and virtual objects with information processes taking into account their mutual relations. In solving these problems, this study used the results of the works of prominent authors, including D. Gordon [4], J. Zahman [5], K. Lockira [6], in the field of application of topos models and category theory - R. Goldblatt [7], Z. Zhang [8], W. J. Xie [8], Y. I. Yang [8], G. Sun [8], Y.-Q. Gao [8] et al. [9-11].

\section{Interaction of Considered Objects of Agreement Processes using Category Theory}

One of the fundamental concepts of the methodology of designing information systems is the concept of the life-cycle of its software, which is regulated by a number of normative documents. The main ones are international standards 
ISO/IEC 12207 and ISO/IEC 15288. The software lifecycle structure is divided into three process groups: core, support, and organizational processes, each with specific tasks, inputs, solution methods, and results. In the very life-cycle of the software, the results of the subsequent stage may necessitate a change in the solutions of earlier stages, i.e. a recursion component is present. The following categorical approach makes it possible to describe all processes under administrative regulations for absolutely all stages using the Chomsky hierarchy. As a result, we come to structuring the relations of objects, in the form of functional relations. With further allocation of objects inside large objects, we get reduction of unstructured part in them and increase of the general structured part of the subject area according to certain rules, which allows to maximize traceability and identification in automated processes [12] up to the whole chain of relations and a specific performer. The properties of ambivalence of relationships - transactions between virtual objects in the information environment are determined both by the specific objects and by the properties of the environment itself.

Using the formalization proposed in $[12,13]$ ISO/IEC 15288 "System Engineering" each stage can be represented as a process category. When considering the agreement process, causes and consequences can be used symmetrically.

The state of the object as a whole in the investigated category can be defined in terms of its "mixed" real and virtual states (Figure 1) [14].

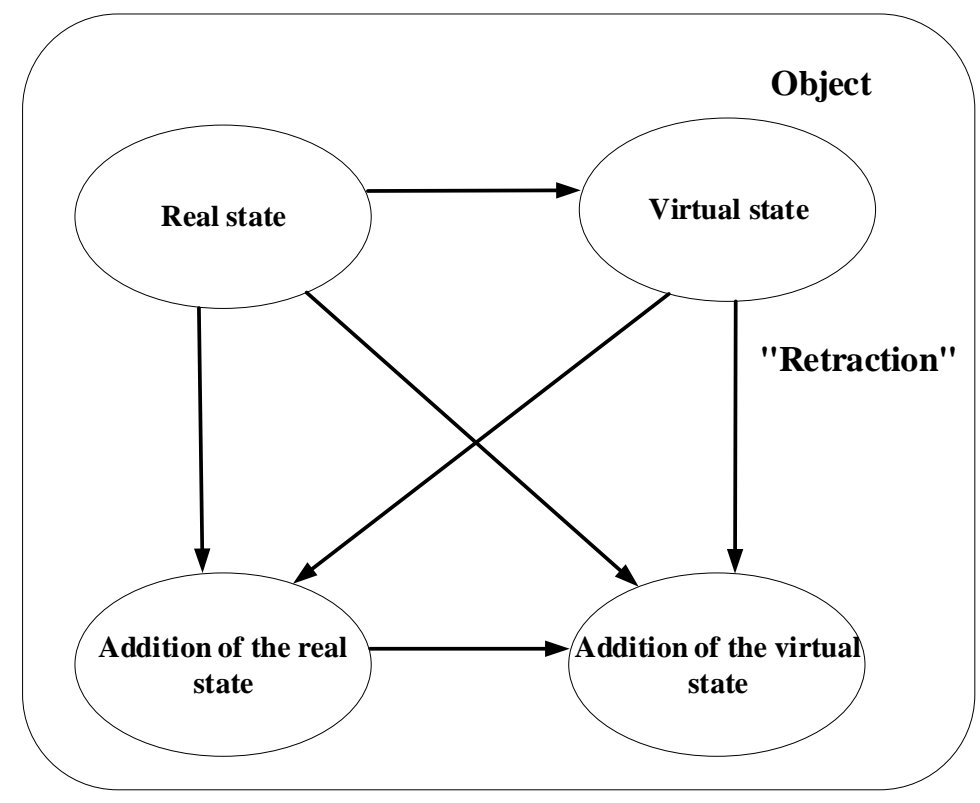

Figure 1

"Mixed" state of the object (similar to cubit) 
Applying the rules of the international standard of system engineering ISO/IEC/IEEE 15288, we can formalize the main components, and accounting objects can be represented by different categories of information objects, related relations, with the relations between virtual objects and real objects expressed by the symbolic model of the data warehouse and define the structure [14].

The system goes through certain stages during its life (the life-cycle (LC) model should consist of stages) [15]. Phase - a period within the LC system that refers to the status of the system description or to the system itself. The stages are associated with significant changes in the life of the system, consistent with the passage of milestones in its development throughout the LC. The LC model may include one or more stage models and is assembled as a sequence of stages that may overlap or repeat depending on the application of the system in question, its size, complexity, varying needs and capabilities. Life-cycle stages form the structural basis for detailed modeling of life-cycles of the system using typical processes of its life-cycle. Each stage represents meaningful progress and achievement of planned stages of system development throughout the entire lifecycle and gives rise to critical decisions regarding inputs and outputs. These solutions are used by organizations to address uncertainties and risks directly associated with costs, timelines, and functionality in creating or implementing the system. In this way, the stages provide the organization with a structure of works, in which the management of the enterprise has a high ability to review and monitor the project and technical processes. The relationship between life-cycle processes can be represented as a diagram in Figure 2 [15].

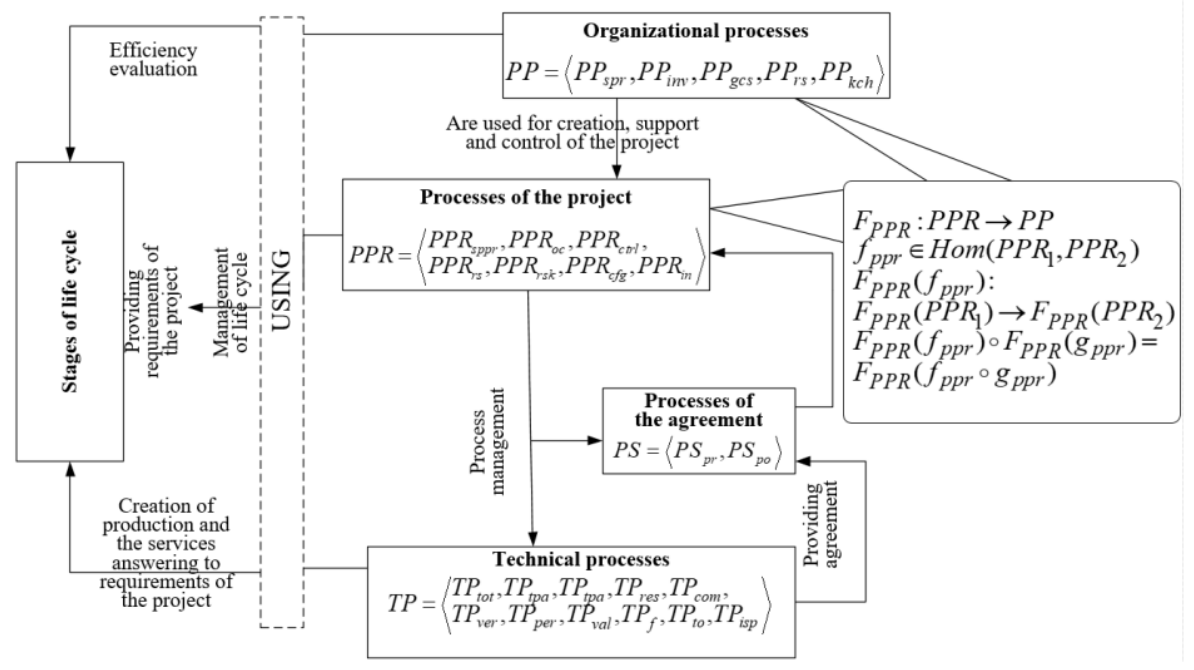

Figure 2

The scheme of communications between processes of life-cycle of system 
The formula is presented as an example of the relationship between technical processes:

$$
\begin{aligned}
& T P=<T P_{t o t}, T P_{t p a}, T P_{t p a}, T P_{r e s}, T P_{c o m}, T P_{v e r}, T P_{p e r}, T P_{v a l}, T P_{f}, T P_{t o}, T P_{i s p}> \\
& T P_{t o t}=\left\{T P_{t o t}^{1}, T P_{t o t}^{2}, T P_{t o t}^{3}\right\}, \ldots, T P_{i s p}=\left\{T P_{i s p}^{1}, T P_{i s p}^{2}, T P_{i s p}^{3}\right\}
\end{aligned}
$$

Note that formula 1 defines the relationship between technical processes as an ordered set.

Thus, technical processes form, a class of objects, for each pair of objects of which and $T P_{2}$ a plurality of morphisms $\operatorname{Hom}\left(T P_{1}, T P_{2}\right)$ are defined, for each pair of which (morphisms), for example $g_{T P} \in \operatorname{Hom}\left(T P_{1}, T P_{2}\right)$ and $f_{T P} \in \operatorname{Hom}\left(T P_{2}, T P_{3}\right)$ their composition $g_{T P} \circ f_{T P} \in \operatorname{Hom}\left(T P_{1}, T P_{3}\right)$ is defined. Technical processes form a category of sets [16].

The relationship between the life-cycle processes of the system can be represented as a diagram in Figure 1, where:

$$
\begin{aligned}
& \text { - organizational processes }-P P=\left\langle P P_{s p r}, P P_{i n v}, P P_{g c s}, P P_{r s}, P P_{k c h}\right\rangle ; \\
& \text { - project processes - } P P R=\left\langle P P R_{s p p r}, P P R_{o c}, P P R_{c t r l}, P P R_{r s}, P P R_{r s k}, P P R_{c f g}, P P R_{i n}\right\rangle ; \\
& \text { - technical processes } \begin{array}{l}
T P=<T P_{t o t}, T P_{t p a}, T P_{t p a}, T P_{r e s}, T P_{c o m}, T P_{v e r}, T P_{p e r} ; \\
\left., T P_{v a l}, T P_{f}, T P_{t o}, T P_{i s p}\right\rangle
\end{array} \\
& \text { - agreement processes - } P S=\left\langle P S_{p r}, P S_{p o}\right\rangle .
\end{aligned}
$$

From the studies carried out $[15,17,18]$ we can conclude that there is a display of categories of sets, preserving the structure of these categories-sets. I.e. the relationship between life-cycle processes, in light of the provisions of the system engineering methodology, is described by functionaries that match an object of one category with an object of another category.

Let some object be highlighted and designated by the term. As a rule, a new object is compared to already known objects, and its information model is formed as a set of comparison of information models of previously known objects. At the same time the model of the new subject area for this object will be built on the basis of the subject area of the object that became known first. As a result, knowledge of the subject area including the object will be structured as a set of properties of the first selected object and a sequence of changes of subject areas of subsequent objects. It turns out that previously selected objects are more privileged with respect to subsequent objects of the subject area, because models of these objects are constructed by changing the model of already known objects. At the same time, the sequence of selecting objects is the will of the case. When you try to select the object that is best suited as the first, you find it most 
convenient to use as its image some idealized, average object of that subject area, the model of which is replaced by a set of variables describing the objects of the subject area. Selecting a set of variables to describe subject area objects and selecting valid values for these variables is largely arbitrary. However, it is this choice that will further determine the limits of the applicability of its model [15, 19].

Thus, the agreement process consists of the acquisition process and the delivery process. At the same time the process is symmetrical, the acquiring side can act both sides at the same time. Using the informational approach, to formalize the subject area, are the categories of objects and the relationships between them. That is, the category can be represented by a plurality of subject area objects and a plurality of relationships there between [12].

Consider the basic positions of category theory $[20,21]$ to simulate this process. We will use the following symbols: agreement processes $P S$, acquisition processes PSpr, delivery processes PSpo (Figure 3).

Let's highlight $P S$ the acquisition $P S p r$ and delivery agreement processes PSpo into separate categories and subcategories, which consist of process objectives, process activities, and result [12]. Consider the interaction of subcategories within which information objects result in a communicative triangle by category and individual subcategories.

According to ISO/IEC 15288 "System Engineering," the agreement process consists of acquisition processes and delivery processes [22, 23].

This category $P S$ forms a class of objects with a given relation for any pairs $\left(P S, P S p r_{p r}\right)$ and $\left(P S, P S p o_{p r}\right)$ whose relationships are defined by a set of morphisms: $f_{1}, f_{2}, p_{1}, p_{2}$.

By treating agreement processes $P S$ as interrelated acquisition $P S p r$ and supply processes $P S p o$, the interaction process can be represented by a Cartesian work $g: P S \rightarrow P S_{p r} \otimes P S_{p o}$, where $g$ is the only morphism expressed as a result of the agreement process. Note that these operations comply with the rules of formal logic. 


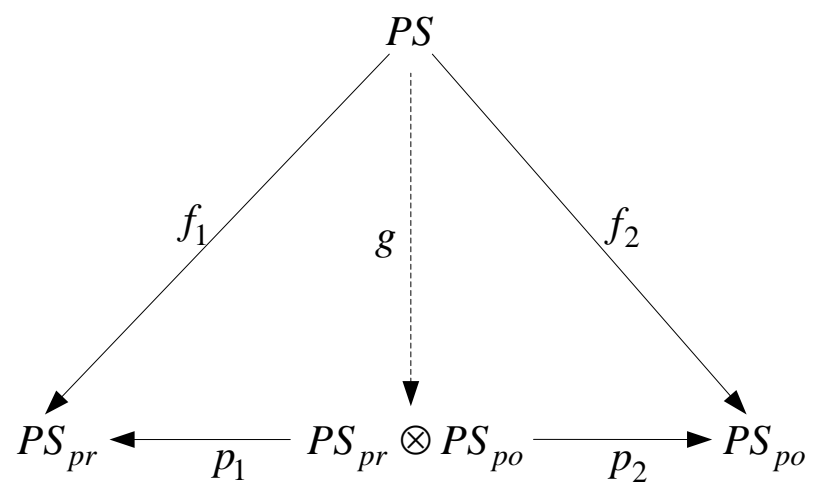

Figure 3

Simplified diagram of the commutative triangle of the agreement process

Thus, the agreement process is formalized as morphisms, and the result of interaction of information objects can be represented by a Cartesian $P S_{p r} \otimes P S_{p o}$ product with morphisms $\quad p_{1}: P S_{p r} \otimes P S_{p o} \rightarrow P S_{p r}^{\prime} \quad$ and $p_{2}: P S_{p r} \otimes P S_{p o} \rightarrow \in P S_{p o}^{\prime}$ such that for any object with $P S$ morphisms $f_{1}: P S \rightarrow P S_{p r}$ and $f_{2}: P S \rightarrow P S_{p o}$ there exists a single morphism $g: P S \rightarrow P S_{p r} \otimes P S_{p o}$ in which a diagram in the form of a commutative triangle is highlighted, where:

$\otimes-$ Cartesian product

$g-$ is the morphism resulting from the process,

Applying the logical convolution of the semantic model of the subject area under study [22], which also contains contradictions relations, we come to their formalization in the form of logical Descartes squares.

Using the research of N. A. Vasilyev [14] of the transition from a logical square to a logical triangle, which reflects the Associative Law of Composition between three vertices "all pairs of judgements-opposites cannot be simultaneously true, but can simultaneously be false," [8], we come to the expediency of applying the Law of Necessary Ashby Variety for two vertices. For example, two of any vertices must have a relationship on the principle of their superposition (nesting, while maintaining and as holistic objects). But simultaneously, relationships between objects can also be "private" (separable), when one object is part of another, then integrity retains one object and the other object is a complement to the first or vice versa, with the superposition property stored for only one object. Thus, to combine associative relations and "private" relations it is possible to use the principle of decomposition of logic triangles, selection of "virtual" objects internal for the considered "large" triangle. It is obvious that additions will appear as new associative (relative to the sides of the triangle) and private as additions. 
These are Rombs (essentially the logical squares of Descartes). This separation logic is well seen on the semantic triangle. To eliminate such potential contradictions, the logical structure of the subject area model should be complemented by cybernetic squares for self-organization. Thus, it is possible to obtain a structure of an open, consistent, self-organized controlled meta-module of an AIC subject area. In the application aspect, this can be realized by a two-step procedure: 1) finding conflicting squares; 2) elimination of detected contradictions by building metamodel with additional squares to cybernetic control system with feedback.

Consider the following separate subcategory: the acquisition process $P S_{p r}$, which consists of three components (Figure 4):

1) Acquisition process purposes $P S_{p r}^{1}=\left\{p r_{1}^{1}\right\}$.

2) Activities in the acquisition process $P S_{p r}^{2}=\left\{p r_{1}^{2}, \ldots, p r_{8}^{2}\right\}$.

3) Result of process of acquisition $P S_{p r}^{3}=\left\{p r_{1}^{3}, \ldots, p r_{7}^{3}\right\}$.

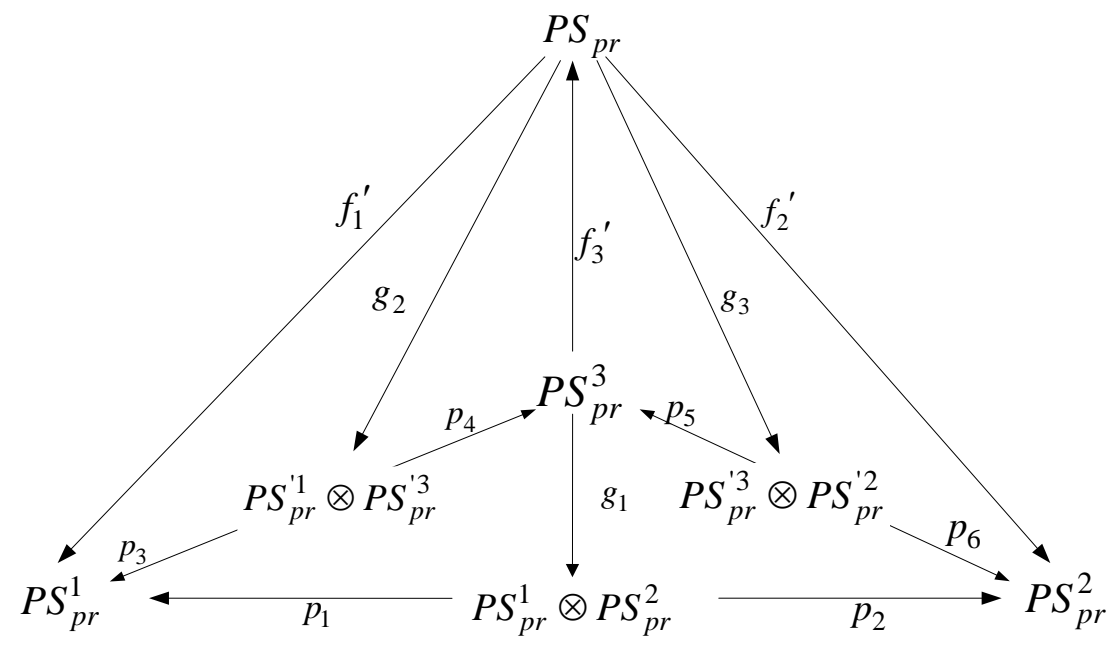

Figure 4

Purchase process commutative triangle

The interaction of information objects $P S_{p r}^{1}$ and $P S_{p r}^{2}$, can be represented by a Cartesian work $P S_{p r}^{1} \otimes P S_{p r}^{2} \quad-$ with morphisms $\quad p_{1}: P S_{p r}^{1} \otimes P S_{p r}^{2} \rightarrow P S_{p r}^{\prime 1}$ and $p_{2}: P S_{p r}^{1} \otimes P S_{p r}^{2} \rightarrow P S_{p r}^{2}$ such that for any information object $P S_{p r}$ with 
morphisms $\quad f_{1}^{\prime}: P S_{p r} \rightarrow P S_{p r}^{1}$ and $\quad f_{2}^{\prime}: P S_{p r} \rightarrow P S_{p r}^{2}$, there is a single morphism $g_{1}: P S_{p r} \rightarrow P S_{p r}^{1} \otimes P S_{p r}^{2}$ which is defined by a commutative diagram.

Similarly, the interaction of objects with morphisms is considered, $p_{3}: P S_{p r}^{\prime 1} \otimes P S_{p r}^{\prime 3} \rightarrow P S_{p r}^{\prime \prime 1}$ and $p_{4}: P S_{p r}^{\prime 1} \otimes P S_{p r}^{\prime 3} \rightarrow P S_{p r}^{\prime \prime 3}$ in which for any object $P S_{p r}$ with morphisms $f_{1}^{\prime}: P S_{p r} \rightarrow P S_{p r}^{1}$ and $f_{3}^{\prime}: P S_{p r} \rightarrow P S_{p r}^{3}$, there is a single morphism $g_{2}: P S_{p r} \rightarrow P S_{p r}^{1} \otimes P S_{p r}^{\prime 3}$ that is defined by a commutative diagram.

In the same way, the interaction of objects $P S_{p r}^{\prime 3} \otimes P S_{p r}^{2}$ with morphisms $p_{5}: P S_{p r}^{\prime 3} \otimes P S_{p r}^{\prime 2} \rightarrow P S_{p r}^{\prime 3}$ and $p_{6}: P S_{p r}^{\prime 3} \otimes P S_{p r}^{\prime 2} \rightarrow P S_{p r}^{\prime 2}$ such that for any object $P S_{p r}$ with morphisms $f_{2}^{\prime}: P S_{p r} \rightarrow P S_{p r}^{2}$ and $f_{3}^{\prime}: P S_{p r} \rightarrow P S_{p r}^{3}$, there is a single morphism $g_{3}: P S_{p r} \rightarrow P S_{p r}^{\prime 3} \otimes P S_{p r}^{\prime 2}$ that is defined by a commutative diagram.

As a result, the acquisition process can be represented as a Cartesian product of the following projections:

$$
P S_{p r}=\left(P S_{p r}^{1}\right)^{\prime} \otimes\left(P S_{p r}^{2}\right)^{\prime} \otimes\left(P S_{p r}^{\prime 1}\right)^{\prime \prime} \otimes\left(P S_{p r}^{\prime 3}\right)^{\prime} \otimes\left(P S_{p r}^{\prime 3}\right)^{\prime \prime} \otimes\left(P S_{p r}^{\prime 2}\right)^{\prime \prime}
$$

Similarly, the interaction of delivery process objects is discussed. The result can be represented as a Cartesian product, and if the result is an independent quantity, it can be intermediate. This allows you to identify the dependence of a recursive point on one another. In other words, if the goal changes, you can predict the result, and information objects are represented as categories, and the ratio of the transition from one stage to another is represented as morphisms.

The pattern of forming a multiple Descartes square for the subject area under study and their decomposition in terms of formal logic and category theory is shown in Figure 5.

When considering agreement processes where the acquisition process $P S_{p r}$, the delivery process $P S_{p o}$, each presented as categories, object interactions can be represented by a set of Cartesian works:

$$
\begin{aligned}
& P S_{p r} \otimes P S_{p o}=\left\langle P S_{p r}^{1} \otimes P S_{p r}^{2}, P S_{p r}^{\prime 1} \otimes P S_{p r}^{\prime 3}, P S_{p r}^{\prime 3} \otimes P S_{p r}^{\prime 2}, P S_{p o}^{1} \otimes P S_{p o}^{2}\right. \\
& \left.P S_{p o}^{\prime 1} \otimes P S_{p o}^{\prime 3}, P S_{p o}^{\prime 3} \otimes P S_{p o}^{\prime 2}\right\rangle .
\end{aligned}
$$




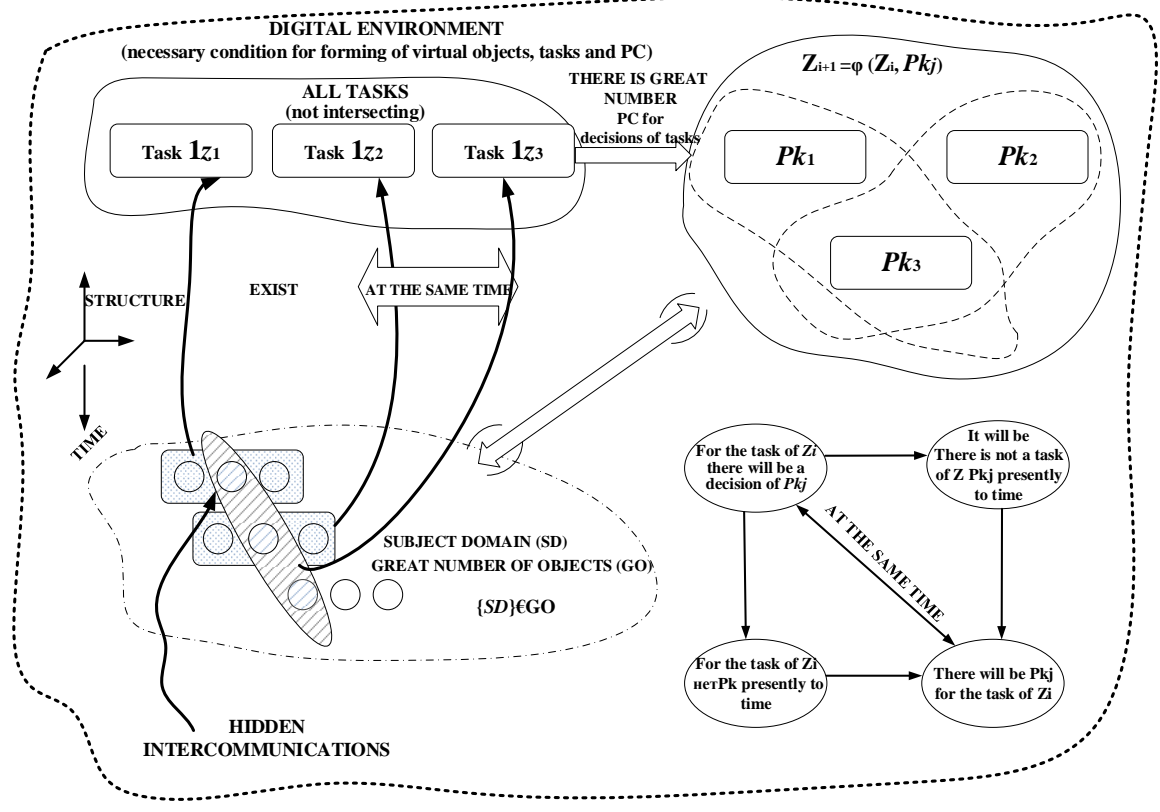

Figure 5

A scenario diagram for forming a multiple Descartes square and decomposing them

Obviously, the agreement process in this case can be represented by the formula:

$$
P S=\left\langle\left(P S_{p r} \otimes P S_{p o}\right),\left(P S_{p o} \otimes P S_{p r}\right)\right\rangle .
$$

Similarly, the acquisition process $P S_{p r}$ can be represented by the formula:

$$
\begin{aligned}
& P S_{p r}=\left\{\left\langle\left(P S_{p r}^{1} \otimes P S_{p r}^{2}\right),\left(P S_{p r}^{2} \otimes P S_{p r}^{1}\right)\right\rangle,\left\langle\left(P S_{p r}^{1} \otimes P S_{p r}^{3}\right),\left(P S_{p r}^{3} \otimes P S_{p r}^{1}\right)\right\rangle,\right. \\
& \left.\left\langle\left(P S_{p r}^{3} \otimes P S_{p r}^{2}\right),\left(P S_{p r}^{2} \otimes P S_{p r}^{3}\right)\right\rangle\right\},
\end{aligned}
$$

And the delivery process $P S_{p o}$ with the formula:

$$
\begin{aligned}
& P S_{p o}=\left\{\left\langle\left(P S_{p o}^{1} \otimes P S_{p o}^{2}\right),\left(P S_{p o}^{2} \otimes P S_{p o}^{1}\right)\right\rangle,\left\langle\left(P S_{p o}^{1} \otimes P S_{p o}^{3}\right),\left(P S_{p o}^{3} \otimes P S_{p o}^{1}\right)\right\rangle,\right. \\
& \left.\left\langle\left(P S_{p o}^{3} \otimes P S_{p o}^{2}\right),\left(P S_{p o}^{2} \otimes P S_{p o}^{3}\right)\right\rangle\right\} .
\end{aligned}
$$

Thus, the interaction between different categories can be described as a functionary based on the Cartesian works presented above. 
In a category $P S_{p r}$, any object will appear in the category objects $P S_{p o}$ that follow:

$$
f \in \operatorname{Hom}\left(P S_{p r}, P S_{p o}\right) \quad\left\{f \in \operatorname{Hom}\left(P S_{p r}^{1}, P S_{p o}^{1}\right), f \in \operatorname{Hom}\left(P S_{p r}^{2}, P S_{p o}^{2}\right)\right. \text {. }
$$

$$
\left.f \in \operatorname{Hom}\left(P S_{p r}^{3}, P S_{p o}^{3}\right)\right\} \text {. }
$$

It also describes how to display objects in a category $P S_{p o}$ :

$$
\begin{aligned}
& g \in \operatorname{Hom}\left(P S_{p r}, P S_{p o}\right) \quad\left\{g \in \operatorname{Hom}\left(P S_{p r}^{1}, P S_{p o}^{1}\right)\right. \\
& g \in \operatorname{Hom}\left(P S_{p r}^{2}, P S_{p o}^{2}\right) \\
& \left.g \in \operatorname{Hom}\left(P S_{p r}^{3}, P S_{p o}^{3}\right)\right\}
\end{aligned}
$$

Similarly, the "Cartesian product" is described, because we will consider it as a new object:

$$
\begin{aligned}
& f \in \operatorname{Hom}\left(P S_{p r} \otimes P S_{p o}, P S_{p o}\right), \\
& g \in \operatorname{Hom}\left(P S_{p o}, P S_{p o} \otimes P S_{p r o}\right), \\
& f \circ g \in \operatorname{Hom}\left(P S_{p r} \otimes P S_{p o}, P S_{p o} \otimes P S_{p r o}\right) .
\end{aligned}
$$

Thus, we get that objects in categories are determined by interaction of functionators. Here we can use the Descartes square, where the composition of the above two processes $g$, indicated, is a direct mapping of the given agreement process to the result. The result of the process can be considered as a composition of the product of goals and activities. It turns out to be another triangle. At the same time, the goal cannot be achieved in one way only. Applying the principle of the necessary diversity of the Ashby [7], according to which, there cannot be the same relations between objects, there must be additional ways to achieve results, we get additional squares in the diagram. In other words, the Descartes square will be determined by the Ashby principle of necessary diversity [2]. These charts satisfy the provisions of the topos, which allows to infer the dependence of, for example, this point on another, by changing the result, for this purpose we determine what needs to be changed for the purposes that in the activity, or we do not change the goals, but only the activity.

It is obtained from the parameters described above, the process can be considered in the form of a combined process and for it the obtained conclusions are fair. That is, the agreement process can be represented by a hierarchical set of nested processes. the dependence of $y=f$ is fair $(x, y)=>y=f(x, f(x, y))$, the selfsimilarity of large-scale invariancy turns out (fractals). In other words, the software system has many variants of development, and the state of the system is determined by the place in which it is currently located. 
The presented rule of synthesis of self-organizing structure of agreement process is based on decomposition - composition of objects-processes by logical addition of fractals in the form of commutative triangles into squares of Descartes and further into Cartesian polyhedron.

When designing a software complex, we will divide it into categories of objects from which it will consist. Given that fractal relations can be established between them. We can understand that once established relation between categories in the form of a functionary, will determine functionary relations. We get many functionaries, which we highlight as a separate category. For instance, relations between fractal elements are built at the level of categorical relationship [3, 18].

Thus, sub-objects constituting a fractal can be described as functionary relationships, i.e. elements of different categories belong to each other via a functionator. As a result, the categories are related to each other by a selfsimilarity relation for a particular subject area.

Fractal in this case acts no geometric place of points, but as a categorical fractal. We will understand the certain categorical object connected by some similarity as a fractal, the similarity is connected by versatility, multi-hierarchy, self-similarity. In terms of category theory, fractal properties will satisfy certain conditions. In this case, each object is a software module, the lower the chain, the greater the number of relationships (Figure 6).

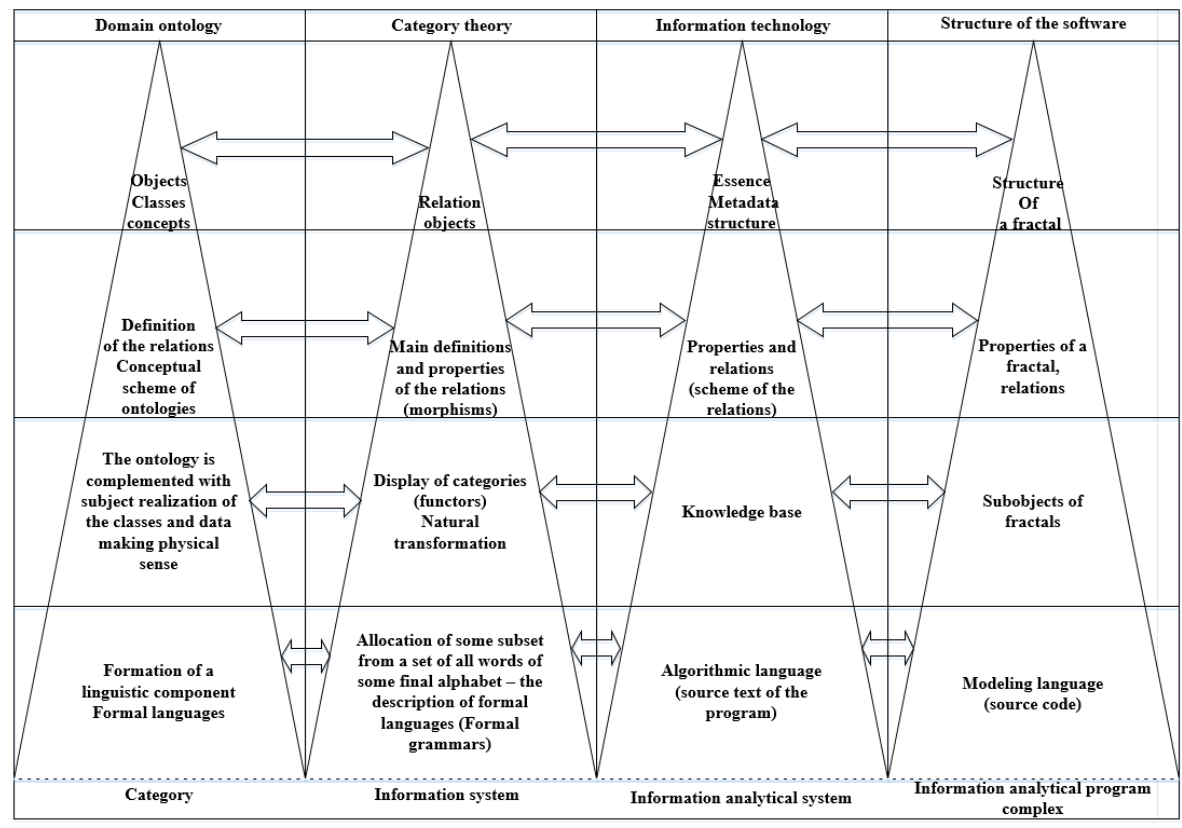

Figure 6

Diagram of relationships between different object categories 
The software is built modulo; this is called a communication vector. We propose the following method: the method of building relationships for further software development and design, i.e. relationships between modules $[7,15]$.

\section{Polycubic Model of Data}

Based on the identified patterns and for ease of presentation of information, it is advisable to present it in the form of multidimensional cubes, the dimensions of which will be the values of attributes of subject areas. Given the constant expansion of the data set, together with the preservation of control, we come to the possibility of building a complex of related registers (in time), including a lot of data received from the outside, i.e. the constant appearance of new virtual objects, which at the next iteration of the system construction become internal. We can talk about the digital twin model control subsystem. The principle of multidimensional models is based on the OLAP concept. OLAP technologies are focused on complex analytical tasks. Figure 7 shows a polycubic olap model for a complex analytical task built by the user. The OLAP model consists of data sources, DataStore, OLAP cubes, and result presentation [24, 25, 28]. The characteristics of the OLAP model include: the power, dimension, volume of the hypercube, where the power of the hypercube is the product of the power of its measurements, and the volume of the hypercube is the product of the power of the cube and the number of indicators.

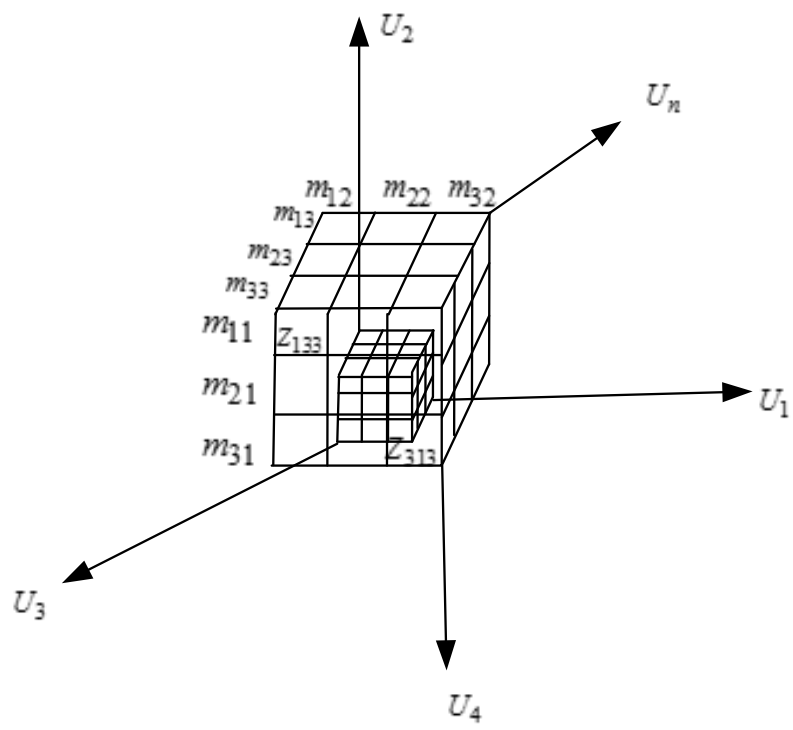

Figure 7

Building a Cube Based on Polycubic Data Organization 
$U=\left\{U_{1}, U_{2}, U_{3}, U_{4}, \ldots, U_{n}\right\}$ - set of measurements of a polycube;

$M_{U_{i}}=\left\{m_{1 i}, m_{2 i}, m_{3 i}, \ldots, m_{s i}\right\}-i=1, \ldots, n-$ set of tags of measurement $U_{i}$;

$M=M_{u_{1}} \cup M_{u_{1}} \cup \ldots \cup M_{u_{n}}$ - set of tags of a polycube;

$Z=\left\{Z_{123}, Z_{223}, \ldots, Z_{\text {mиi }}\right\}-$ a plurality of cells with measurements of a polycube.

In polycubic data organization, a multidimensional database is represented by several hypercubes, with different dimensions and dimensions. Each cell is a category, the relationship between them is functional. As a result, we can use different intersection points for different needs. For example, the time vector can be applied to call a module of this year or a previous one. This ensures traceability and repeatability of the output results. When considering the vector "competences," it is possible to pick, regardless of training, any new set of competences.

Using a polycubic data model will allow to define hypercubes with different dimensions and with different measurements as their faces [26].

Let's consider the hypercube construction option, for which we will produce the necessary conversion of the data stored in the database tables. For example, in order to improve performance when building a hypercube, we find unique elements stored in columns that will be measurements of the hypercube. Records that have the same dimension values are pre-aggregated. To create hypercube slices, you must define the coordinates (actual measurement values) for the table entries and define the entries. Actions are divided into two stages - according to the given classification of attributes. For identification and functional attributes, it is possible to create a table of unique values on the basis of which the measurement will be built. Depending on the type of business processes to be integrated, we define an integration rule. For formalized attributes, based on the set semantic dependencies between accounting object types, define an integration rule.

The transformations are schematically shown in Figure 8. We define keywords (phrases), draw up a table of the accuracy of variants of their combinations satisfying the semantic rule (by the function of belonging) and as in the case of formalized attributes we build a table of unique values. Instead of a single table, we get the equivalent of a normalized database. Define the coordinates for the measurement values by renumbered the element values. Lists of measurement values are pre-sorted alphabetically within a single dimension [15]. 


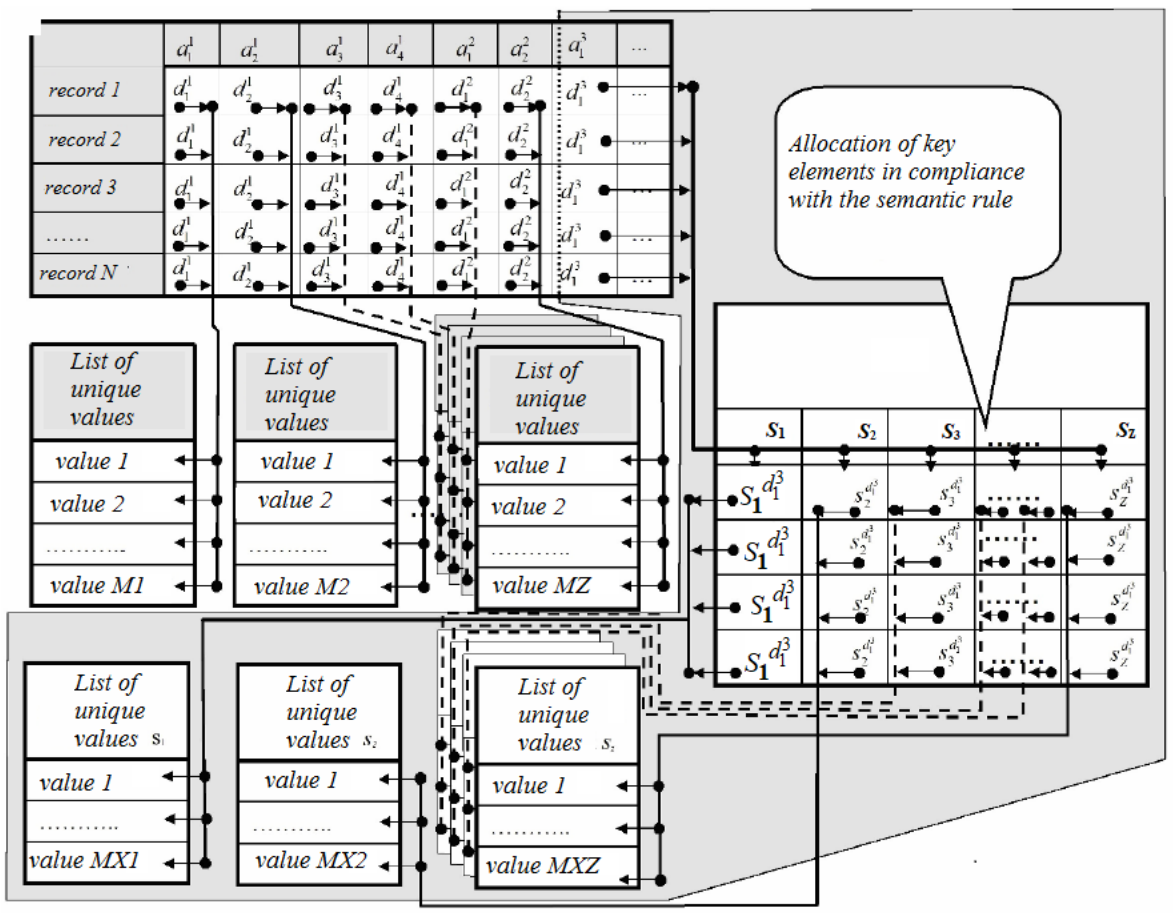

Figure 8

Example of formation Olap-cub

\section{Information Support of the Subject Area under Consideration based on the Data Warehouse}

Using theoretical-multiple models of agreement processes, an information and analytical system (IAS) scheme based on data repositories was built (Figure 5).

IAS is characterized by the following parameters: use of various database management systems (Oracle, MySQL, etc.), modern storage tools (Data Warehouse), application of Online Transaction Processing (OLTP), On-line Analytical Processing (OLAP), Data Mining, etc.

As a result of IAS use, users (decision-makers, analysts, experts) receive an effective tool for selection, analysis of candidates for personnel reserve, as well as generation of data on personnel reserve, presented in tabular and graphical form. This IAS provides centralized collection, storage, updating, structuring, systematization, integration, processing and consolidated analysis of diverse data of candidates of personnel reserve $[16,17]$. 
In IAS subsystems comply with the following principles:

- Razvivayemost;

- Construction blochnost;

- Certain independence of individual subsystems and their databases (DB);

- Correction of functional part, etc.

The main data for the DB is the candidate data directory, which has full information about the candidate, the name of the table in which the key figures are stored, the key figure group code to which the key figure belongs, the key figure to the sub-indicators, the key figure unit code, the frequency of the key figure presentation and the field with the comment for the key figure. The knowledge base contains rules of the algorithm of the program operation, described by means of categories of sets.

External sources of data are: state authorities (Ministries of Labor and Social Protection, local administration of the city and district, etc.), cooperating organizations (employment centers, universities, enterprises, etc.), higher authorities (holdings, corporations, etc.).

The use of a formal description of information objects in the language of category theory helps to increase the formality of the subject area, opening up new opportunities to identify problems [26, 27].

When forming storage of data, the separate objects expressed by categories of users, categories of program modules can be considered, the relations between them will be defined by functors. This allows data and knowledge to be stored in a single place. Thus, the problem of data and knowledge separation in processing and transmission is solved.

\section{Conclusions}

Invention proposes a rule for synthesis of a self-organizing structure, of an agreement process, based on sequential decomposition - composition of process objects in the form of commutative triangles into Descartes squares and further into Cartesian polyhedron. We can say that for all elementary structures the rules of formal logic, parametric control of the state of processes are preserved. The structure of the agreement process itself is synthesized by the scheme above. This makes it possible to apply decentralization-based technologies - the use of blockchain, and eventually the development of a fully decentralized platform on which business processes are carried out. Taking into account the abovementioned studies, built on a strict mathematical basis, the prerequisites for creating a single communication language are created. Moreover, all this is represented as a system of equations derived from a formal software model. This method of transition to a quantitative mono-variant description of information processes and the application of the requirements of ISO / IEC 15288 standard, 
allows one to link each stage of the life-cycle of the developed information analytical system by introducing recursion, recursively searching for the optimal solution of subtasks, performing a similar sequence of actions. The Olap cube was built on the basis of polycubic data organization.

The Application of the method of semantic differentials of C. Osgood, for processing and presentation of information, in relation to stages of the process under consideration, provides the possibility for building the required functions of belonging.

\section{Acknowledgement}

The study is conducted with financial support from the Ministry of Education and Science of the Russian Federation as part of the basic part of the state assignment to higher education educational institutions \# FEUE-2020-0007.

\section{References}

[1] Filatova E. V.: Investments in human capital at small businesses [Text]: thesis abstract of Cand. Econ. Sciences, M: 2010, 177 p.

[2] Bazarova T. Yu., Eremina B. L.: Personnel management. Textbook for high schools / Ed. T. Yu Bazarov, B. L. Eremina. - $2^{\text {nd }}$ ed, changed and added. M: UNITY, 2007, 560 p.

[3] Kovács, G. L., Petunin, A. A.: An information technology view of manufacturing automation, Product life-cycle management, Pollack Periodica, Vol. 11, No. 2, 2016, pp. 3-14

[4] Zachman, J. A.: A Framework for Information System Architecture, IBM System Journal, Vol. 26, No. 3, 1987, 276 p.

[5] Lokir K., Gordon O.: Project Management: Stages of Excellence, Publishing House, 2008, 352 p.

[6] D. Gianni, A. D’Ambrogio, A. Tolk (eds.): Modeling and simulation-based systems engineering handbook, CRC Press, London, 2014, 513 p.

[7] Goldblatt R. Toposa. Category analysis of logic. Moscow: Mir, 1983, 486 p.

[8] Z. Zhang, W.-J. Xie, Y. I. Yang, G. Sun, Y.-Q. Gao: Simulation studies of the self-assembly of halogen-bonded sierpiński triangle fractals, Wuli Huaxue Xuebao. Acta Physico, Chimica Sinica, Vol. 30, No. 3, 2017, pp. $539-547$

[9] Mabrok M. A., Ryan M. J.: "Category theory as a formal mathematical foundation for model-based systems engineering", Appl. Math. Inform. Sci., Vol. 11, No 1, 2017, pp. 43-51

[10] Uhlemann T. H.-J., Steinhilper C. L. R., Steinhilper R.: The Digital Twin: Realizing the Cyber-Physical Production System for Industry 4.0. Procedia 
CIRP, Part of special issue: The $24^{\text {th }}$ CIRP Conference on Life-cycle Engineering. Ed. by S. Takata, Y. Umeda, S. Kondoh, Vol. 61, 2017, pp. 335-340, DOI: 10.1016/j.procir.2016.11.152

[11] Baumester D., Jekert A., Cajlinger A.: Fizika Kvantovoj Informacii. M.: Postmarket, 2002, 376 p.

[12] G. G. Kulikov, V. V. Antonov, D. V. Antonov, F. F. Shingarev: Method of subject-oriented classification and system modeling of weakly formalized information flows in industrial automation systems, Bulletin of SUSU. A series of "Computer technologies, management, radio electronics", Vol. 16, No. 2, 2016. pp. 116-130, DOI: 10.14529 / ctcr160212

[13] Rechkalov AV, Kulikov GG, Antonov VV, Artyukhov AV.: Development of a formal model of the production process for the organization of project and production management with the use of intellectual KIC, Bulletin of PNIPU. Electrical engineering, information technology, control systems, No 11, 2014, pp. 34-54

[14] Vasilyev N. A.: Imaginary logic. Selected works, M: Nauka, 1989, 264 p.

[15] Cordon, O., Herrera F.: General study on genetic fuzzy systems, Genetic Algorithms in engineering and computer science, 1995, pp. 33-57

[16] Artemyev, S. A., Zamay S. S., Pitenko A. A.: GIS the designer with means of the analysis of the information and analytical systems of, Computing technologies, Messenger TREASURY, Vol. 9, No. 3(42), 2004, pp. 188192

[17] Massel L. V. Kovalyov: Fractal approach to structuring knowledge and examples of its application, design Ontology, Vol. 6, No. 2 (20), 2016, 149 p.

[18] Kovalev S. P.: Teoretiko-category approach to design of programm systems, base. and approx. maty., Vol. 19, No 3, 2014, pp. 111-170

[19] E. E. Akimkina, A. E. Abbasov.: Analysis of the tools of information systems for processing multidimensional data, Information and Technology Bulletin, 2015, pp. 61-75

[20] Kovalyov S. P.: Category of computing systems, International conference "Algebra and Logic: Theory and Applications". Theses of reports. Krasnoyarsk: SFU, 2013, pp. 64-66

[21] Kulikov, G. G. Antonov V. V., Antonov D. V.: Theoretical and applicationoriented aspects of creation of models of information systems of, LAP LAMBERT Academic Publishing GmbH \& Co.KG, Germany, 2011, 134 p.

[22] G. G. Kulikov, V. V. Antonov, M. A. Shilina, A. R. Fahrullin: Mathematical and software for the construction and implementation of object-oriented IUS from the conditions of identification and traceability, Journal of the UURSU. Series "Computer Technologies, Control, Radio 
Electronics.", Vol. 16, No. 3, 2016, pp. 143-151, DOI: $10.14529 /$ ctcr 160316

[23] ISO/IEC/IEEE 15288:2015 System and software engineering - System lifecycle processes

[24] Kovács, G., Yussupova, N., Rizvanov, D.: Resource management simulation using multi-agent approach and semantic constraints, Pollack Periodica, An International Journal for Engineering and Information Sciences, Vol. 12, 2017, pp. 45-58 https://doi.org/10.1556/606.2017.12.1.4

[25] Abdulnagimov A., Ageev G.: Neural Network Technologies in Half-Time Modeling: Principles of Implementation of Digital Dual GTS, Bulletin USATU, Ufa, Vol. 23, No. 4 (86), 2019, pp. 115-121

[26] Kulikov, G. G., Antonov V. V., Savina A. A.: Information and Analytical System Based on Knowledge of Population Registration, UGATU: Scientific journal of Ufa state aviation technical University, Vol. 10, No. 2(27), 2008, pp. 60-67

[27] Antonov V. V. Kulikov G. G., Antonov D. V.: Formalization of subject domain with use of the tools supporting standards, UGATU: Scientific journal of Ufa state aviation technical University, Vol. 16, No. 3 (48), 2012, pp. $42-52$

[28] Yussupova, Nafissa \& Kovacs, George \& Boyko, Maxim \& Bogdanova, Diana: Models and Methods for Quality Management Based on Artificial Intelligence Applications. Acta Polytechnica Hungarica, No. 3, 2016, pp. $45-60$ 